\title{
E-by-e fluctuations of initial conditions in interactions of light, intermediate and heavy nuclei at energies 4 - $200 \mathrm{GeV}$ per nucleon
}

\author{
A.A. Loktionov ${ }^{1, *}, A . K h$. Argynova ${ }^{1, * *}$, and T.N. Kvochkina ${ }^{2, * * *}$ \\ ${ }^{1}$ Institute of Physics and Technology, Satbaev University, Almaty, Kazakhstan \\ ${ }^{2}$ Institute of Nuclear Physics, Almaty, Kazakhstan
}

\begin{abstract}
A better understanding of initial conditions of nuclear interactions is one of the most important heavy ion physics problems. The paper considers these problems in the centrality selected interactions of light $(\mathrm{C}, \mathrm{O}, \mathrm{Ne})-$ (beam) and heavy $(\mathrm{Au}, \mathrm{Pb})-($ beam) ions with light $(\mathrm{C} / \mathrm{N} / \mathrm{O})-($ target $)$ and heavy $(\mathrm{Ag} / \mathrm{Br})$ - (target) nuclei using the data of JINR-AGS-SPS target emulsion experiments with limited statistics. To reveal a possible system structure dependence, the analyses of E-by-E fluctuations on the longitudinal event shape and multiplicity have been performed, vertically and horizontally.
\end{abstract}

\section{Introduction}

A better understanding of initial conditions of nuclear interactions is one of the most important heavy ion physics problems. Interesting results of these studies have been obtained in the new collider and target experiments and considered at recent conferences.

An opportunities to analyse the E-by-E initial state fluctuations has been revealed by ALICE collaboration [1]. For $\mathrm{Pb}-\mathrm{Pb}$ interactions at $\sqrt{s}=2.76 \mathrm{TeV}$, it has been shown that heavy ion events can be classified according to the asymmetry determined from measurements of the neutron spectator energies on both sides of the collision. For Xe-Xe at $\sqrt{s}=5.44 \mathrm{TeV}$ and $\mathrm{Pb}-\mathrm{Pb}$ at $\sqrt{s}=5.02 \mathrm{TeV}$ interactions, the CMS and ALICE comparative analyses of charged particle multiplicities have shown [2-4] that particle production is driven by collision geometry instead of the system size. The SPS NA61/SHINE fixed-target experiment has performed the comparative analyses of the scaled variance of charged hadron multiplicity in proton-proton, Be-Be, and Ar-Sc collisions [5]. A wide range of experiments on the interactions of both light and heavy nuclei in the fixed-target and collider modes is being prepared at NICA collaborations [6]. New LHCb experiments with gas targets [7] have a large potential to probe and characterize the initial state of heavy ion interactions. BNL STAR collaboration has successfully carried out a methodological analysis of interactions Al-Au at $\sqrt{s}=4.9 \mathrm{GeV}$ and $\mathrm{Au}-\mathrm{Au}$ at $\sqrt{s}=4.5 \mathrm{GeV}$ in the framework of the fixed-target configuration of detectors [8].

Thus, to solve the problem of the initial state in high-energy ion physics, we need new instrumental solutions, new experimental data, and new analytical approaches.

\footnotetext{
*e-mail: aaloktion@yandex.kz

**e-mail: argali7@mail.ru

***e-mail: kvochtn@mail.ru
} 
The work has considered fluctuations of the initial conditions in the nuclear interactions as the system size problem of multiparticle dynamics. Our primary goal is to reveal initial fluctuations based on the results of the experiment, with minimal involvement of additional information. For this goal the system size dependence of mutiparticle processes has been investigated in centrality of selected nuclear interactions of light, medium and heavy nuclei at JINR-AGS-SPS energies. The analyses have been performed in variables of multiplicity and pseudorapidity. Fluctuations in the transverse plane have not been considered.

The paper is organized as follows: Sect. 2 provides an introduction to the data processing and the details of the vertical and horizontal estimates of E-by-E fluctuations. Sect. 3 presents the results of the analysis. Summary and outlook are presented in Sect. 4.

\section{Vertical and horizontal estimates E-by-E fluctuations on multiplicity and pseudorapidity}

E-by-E fluctuations are an efficient tool for analyzing multiparticle processes. The classification of the E-by-E data, without summation on the ensemble of events, allows one to carry out a more precise selection of new phenomena. Therefore, E-by-E fluctuation analysis becomes an integral part of modern experiments.

\subsection{Experimental variables}

In order to study the processes of multiparticle dynamics, only the events with high multiplicities were accepted: $N_{s}>1 / 3\left(N_{s}\right)_{\max }$ corresponds to the maximal multiplicity in the experiment. The basic variables of the analyses are as follows:

- The normalized multiplicity of the produced charged particles - $N_{s} /\left(N_{s}\right)_{\max }$, and the normalized sum of the mass of all charged fragment-spectators: $-A_{f} / A_{\max }$. $A_{\max }$ corresponds to the mass of projectile nucleus. The concept of normalized variables allowed us to combine the results of experiments at the energies from JINR to SPS with sufficient accuracy.

- Multiplicity-fragmentation correlations $N_{s} /\left(N_{s}\right)_{\max }-A_{f} / A_{\max }$ between the normalized multiplicity and normalized sum of the mass of all charged fragment-spectators.

- The third and fourth statistical moments of full pseudorapidity distributions to study Eby-E fluctuations for the longitudinal event shape.

\subsection{Centrality estimation}

The measurable estimator of centrality has been proposed [8] and realized [9], using correlations $N_{s} /\left(N_{s}\right)_{\max }-A_{f} / A_{\max }$ between multiplicity and sum of all fast charged, light and heavy, fragment-spectators: proton-spectators, alpha-particle-spectators and heavy mass fragmentspectators. In this approach the number of participant nucleons for projectile nucleus is

$$
N_{\text {part }}(\text { projectile })=A_{\max }-A_{f}
$$

The model of quantitative Glauber nuclear geometry to describe (near) right spherical nucleus collisions was not used. The limited statistics of the data allowed one to select only three intervals of centrality: central, mid-central and peripheral. 


\subsection{The study of E-by-E fluctuations for the longitudinal event shape}

Independent and accurate measurement in each event correlation between charged multiplicity and the sum of all fast charged fragment-spectators open the way for efficient estimation of the E-by-E fluctuations of multiplicity.

The study of the higher statistical moments in each event, the third and fourth statistical moments, of pseudorapidity distribution gives us a clear picture of E-by-E fluctuations for the longitudinal event shape. The fourth statistical moment, Kurtosis $(\mathrm{K})$, measures the sharpness of the distribution relative to a Gaussian. Negative $\mathrm{K}$ implies that the peak is broader than a Gaussian.

The third statistical moment, Skewness (S), in this context "works" as an indicator of accuracy in symmetrical collisions.

\subsection{Vertical and horizontal estimates E-by-E fluctuations on pseudorapidity}

The evolution of experimental fluctuation patterns has been estimated in two directions: vertical and horizontal. In the vertical analysis of E-by-E fluctuations in interactions of each group of nuclei, light-light $(\mathrm{C}, \mathrm{O}, \mathrm{Ne})-($ beam $)+(\mathrm{C} / \mathrm{N} / \mathrm{O})-($ target $)$, intermediate-light $(\mathrm{Si}$, $\mathrm{S})-($ beam $)+(\mathrm{C} / \mathrm{N} / \mathrm{O})$ - (target) and heavy-heavy $(\mathrm{Au}, \mathrm{Pb})-($ beam $)+(\mathrm{Ag} / \mathrm{Br})-($ target $)$, have been compared with each other at a given level of centrality: at central, mid-central and peripheral. In the horizontal analysis of the E-by-E fluctuations in these groups of interactions have been compared with each other at a different level of centrality: from central, via mid-central and to peripheral.

This approach can give a new insight not only on the problem of the system size, but also on the problem of the system structure.

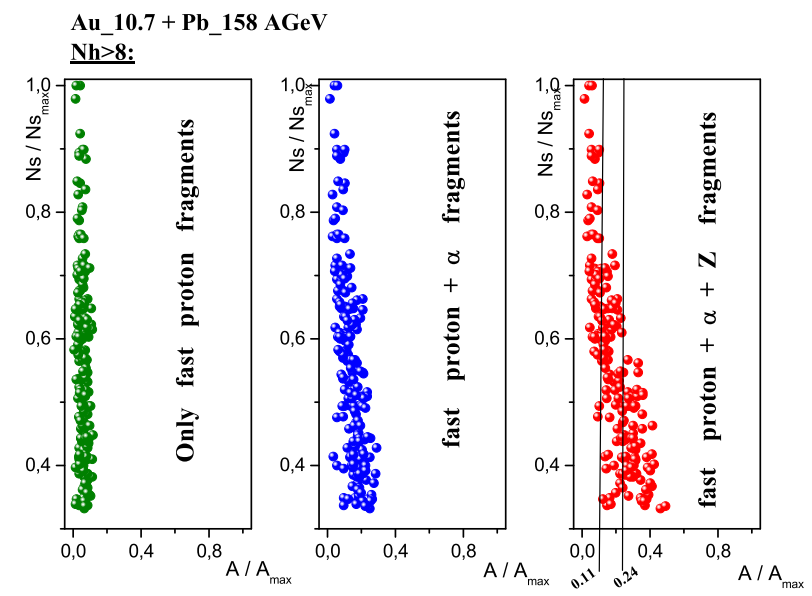

Figure 1. Multiplicity-fragmentation correlations for $(\mathrm{Au}, \mathrm{Pb})+(\mathrm{Ag} / \mathrm{Br})$ interactions

\section{Results}

\subsection{The study of E-by-E multiplicity-fragmentation correlations}

The results of the analyses in the graphical form are presented in Fig. 1 for $(\mathrm{Au}, \mathrm{Pb})$ beam $+(\mathrm{Ag} / \mathrm{Br})-$ target and in Fig. 4 for $(\mathrm{C}, \mathrm{O}, \mathrm{Ne})-($ beam $)+(\mathrm{C} / \mathrm{N} / \mathrm{O})-($ target $)$ interactions. In these figures, different spectators are consistently taken into account in the 

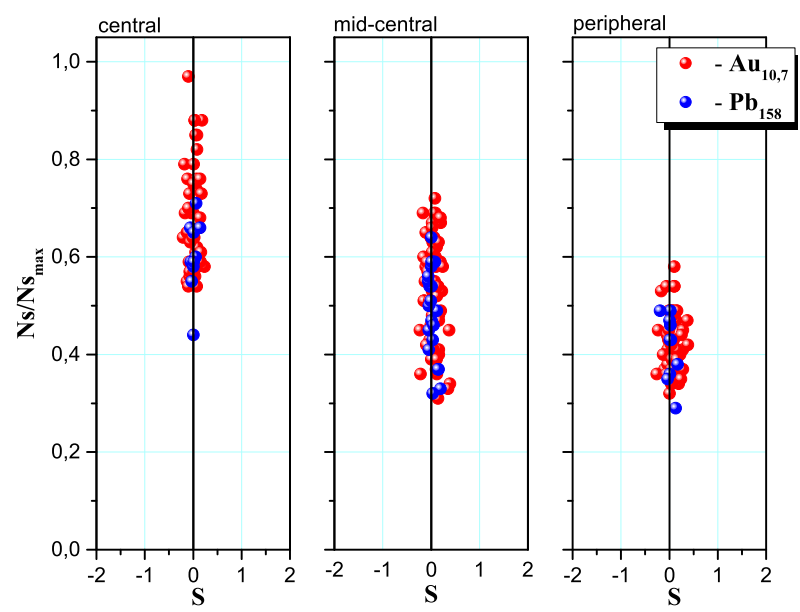

Figure 2. E-by-E accuracy $(\mathrm{S})$ analysis for $(\mathrm{Au}, \mathrm{Pb})+(\mathrm{Ag} / \mathrm{Br})$ interactions
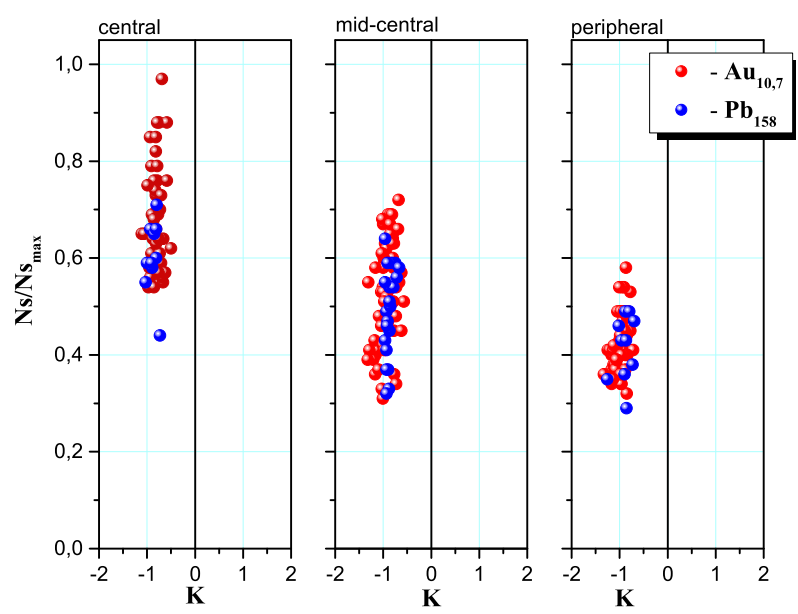

Figure 3. E-by-E Kurtosis $(\mathrm{K})$ analysis for $(\mathrm{Au}, \mathrm{Pb})+(\mathrm{Ag} / \mathrm{Br})$ interactions

left (proton-spectators), middle (proton + alpha-particle-spectators) and right frame (proton+alpha+heavy mass fragment-spectators). All correlation panels have shown centrality intervals with the most central collisions in the top-left, whereas with the most peripheral ones in the bottom-right.

\subsection{The study of E-by-E fluctuations for the longitudinal event shape}

The results of the parallel comparative analyses of vertical and horizontal E-by-E fluctuations of multiple processes for $(\mathrm{Au}, \mathrm{Pb})-$ beam $+(\mathrm{Ag} / \mathrm{Br})-$ target and $(\mathrm{C}, \mathrm{O}, \mathrm{Ne})-($ beam $)+$ $(\mathrm{C} / \mathrm{N} / \mathrm{O})$ - (target) interactions are presented in Figs. 3 and 6. They contain E-by-E analysis of the fourth statistical moment. 


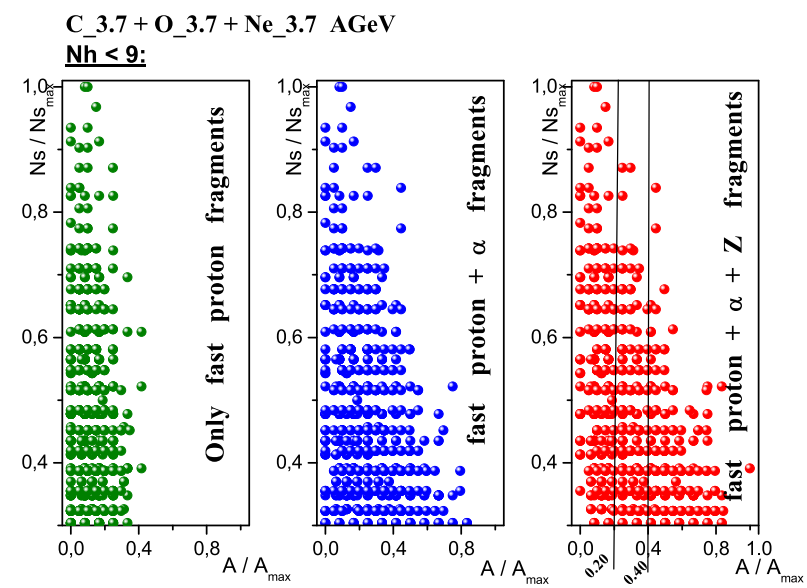

Figure 4. Multiplicity-fragmentation correlations for $(\mathrm{C}, \mathrm{O}, \mathrm{Ne})+(\mathrm{C} / \mathrm{N} / \mathrm{O})$ interactions

The vertical analysis for central interactions has shown that there is a clear trend to the E-by-E fluctuation increasing with system size decreasing. It should be emphasized that this trend continues for all levels of centrality - central, mid-central and peripheral.

The horizontal analysis has demonstrated that in the heavy-heavy collisions the fluctuations for all levels of centrality, central, mid-central and peripheral, are (almost) the same and small. In the light-light collisions the fluctuations for all levels of centrality, central, mid-central and peripheral, are the same and large. These results require a more detailed comparison with theoretical predictions [14].

Therefore, the system size dependence is not reduced to a simple sum of interacting nucleons. The properties of the parent nuclei are projected onto this dependency.

\subsection{The E-by-E accuracy analyses}

The results of the accuracy analyses for $(\mathrm{Au}, \mathrm{Pb})-($ beam $)+(\mathrm{Ag} / \mathrm{Br})-($ target $)$ and $(\mathrm{C}, \mathrm{O}, \mathrm{Ne})$ - (beam) $+(\mathrm{C} / \mathrm{N} / \mathrm{O})$ - (target) interactions are presented in Figs. 2 and 5. They contain E-by$\mathrm{E}$ analysis of the third statistical moment. Here it works as a precision indicator. Analysis of the distributions of the 3rd moment has shown that all variants of the analysis have high accuracy. The mean values of all distributions are close to zero. However, the variance changes significantly. In light-light interactions it is much higher (by $5-10$ times) than in heavy-heavy collisions.

\subsection{Comparison and discussion of the results}

The results of the present work can be compared with the new and recent results of the system size.

\subsection{1}

Strong enhancement of E-by-E fluctuations in the longitudinal event shape and multiplicity for the central collisions of $(\mathrm{C}, \mathrm{O}, \mathrm{Ne})-($ beam $)+(\mathrm{C} / \mathrm{N} / \mathrm{O})-($ target $)$ was interpreted as the sign 
of intrinsic alpha-clustering in light nuclei [11-13]. Indeed, the transition to the virtual alphacluster structure of nuclei sharply violates the spherically symmetric picture of light nuclei. Accordingly, in the interactions of light nuclei the probability of asymmetric collisions of virtual alpha-clusters should increase sharply as well. This assumed to lead to increasing the E-by-E fluctuations for the longitudinal event shape and multiplicity.

Experimentally the small effect of different longitudinal asymmetries, as the result of an unequal number of participating nucleons in the two colliding nucleus, has recently been recognized by ALICE collaboration [1] for $\mathrm{Pb}-\mathrm{Pb}$ interactions at LHC energies.

In this context, it is interesting to note the results of computer simulation for asymmetric collisions of light-heavy nuclei $[16,17]$, in which the alpha-cluster structures of the excited fireball were studied and discussed in detail.

\section{4 .2}

The broad system size studies have been performed on the fixed-target NA61/SHINE experiments at SPS. Recent comparative analysis of fluctuations in p-p, Be-Be, and Ar-Sc collisions has shown [5] that the scaled variance of charged hadron multiplicity changes a little, when going from p-p to Be-Be collisions, and drops dramatically from Be-Be to Ar-Sc interactions. Interpretation of data becomes a huge challenge.

An interesting theoretical comparison of experimental data with popular models (EPOS, PHSD, UrQM D) is performed in [15]. However, the comparison of the experiment NA61/SHINE with the theory for full multiplicity fluctuations has been calculated only for proton-proton and $\mathrm{Pb}-\mathrm{Pb}$ interactions from older NA49 measurements. The forthcoming comparison of theory and experiment for light nucleus-nucleus collisions [15] at SPS energies will be very curios.
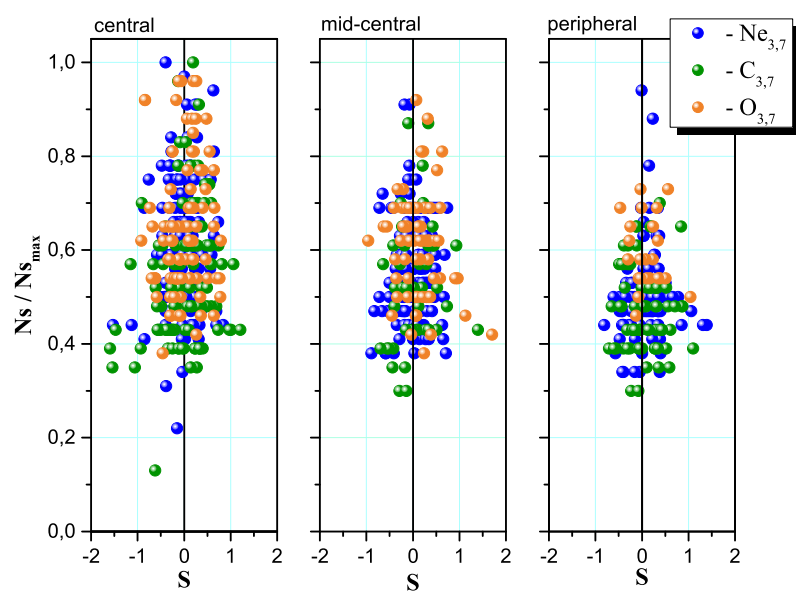

Figure 5. E-by-E accuracy $(\mathrm{S})$ analysis for $(\mathrm{C}, \mathrm{O}, \mathrm{Ne})+(\mathrm{C} / \mathrm{N} / \mathrm{O})$ interactions

\section{Summary and outlook}

The parallel comparative analyses of the E-by-E fluctuations for the longitudinal event shape and multiplicity, vertical and horizontal, have improved understanding of the fluctuating 

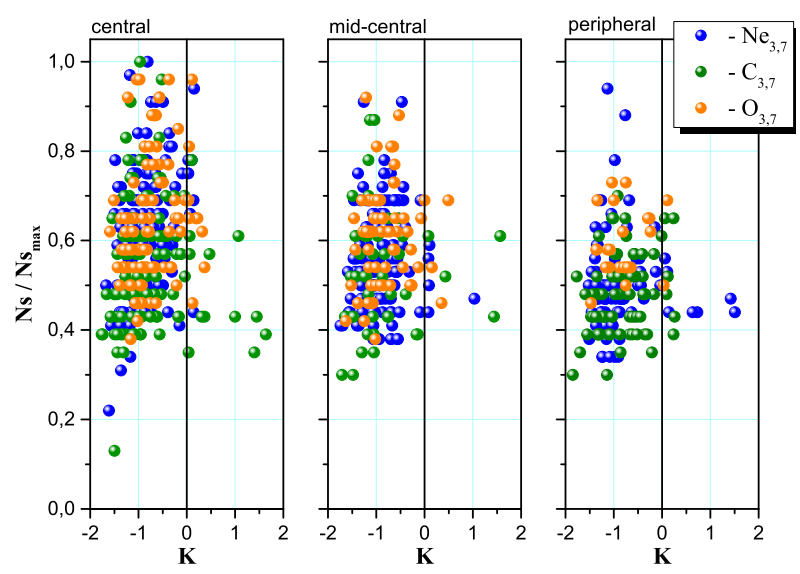

Figure 6. E-by-E Kurtosis $(\mathrm{K})$ analysis for $(\mathrm{C}, \mathrm{O}, \mathrm{Ne})+(\mathrm{C} / \mathrm{N} / \mathrm{O})$ interactions

structures in the initial conditions. The system size dependence is not reduced to a simple sum of interacting nucleons. The properties of the parent nuclei are projected onto this dependency.

These approaches complement the previous results of our research group which have shown that there is a clear trend to the E-by-E fluctuation increasing with system size decreasing for central interactions of light-light, medium-light and heavy-heavy nuclei. The strong enhancement E-by-E fluctuations in the longitudinal event shape and multiplicity for the central collisions of $(\mathrm{C}, \mathrm{O}, \mathrm{Ne})-($ beam $)+(\mathrm{C} / \mathrm{N} / \mathrm{O})-($ target $)$ has been interpreted as the sign of intrinsic alpha-clustering in light nuclei.

In order to make the quantitative inference on the system size and structure dependence for multihadron production processes, the new physical approaches and experiments with high resolution and statistics are needed. There are excellent opportunities of carrying out new experiments on the interactions of both light and heavy nuclei in the fixed-target and collider modes, which are being prepared at NICA collaborations.

\section{References}

[1] S. Acharaya, J. Adam, D. Adamova et al. (ALICE Collaboration), arXiv: 1710.07975

[2] R.Bi (for CMS Collaboration), QM2018 (2018)

[3] B. Kim (for ALICE Collaboration), QM2018 (2018)

[4] G. Feofilov (for ALICE Collaboration), Baldin ISHEPP XXIV (2018)

[5] K. Grebieszkow (for the NA61/SHINE Collaboration), CPOD 2018 (2018)

[6] V. Kekelidze (NICA Collaboration), QM2018 (2018)

[7] M. Vinn (for the LHCb collaboration), arXiv: 1808.08152

[8] Y. Wu (for STAR Collaboration), QM2018 (2018). arXiv: 1807.06738

[9] A.Kh. Argynova, A.Sh. Gaitinov, A.A. Loktionov, Intern. Conf. ICNRP'13, Nuclear and Radiation Physics, Almaty, Kazakhstan (2013)

[10] A.A. Loctionov, A.Kh. Argynova, A.Sh. Gaitinov, Poster I-18, QM2014 (2014). http://indico.cern.ch/event/219436/session/2/

[11] A.A. Loktionov, A.Kh. Argynova, T.N. Kvochkina, Baldin ISHEPP XXIV (2018)

[12] A.A. Loktionov, A.Kh. Argynova et al., Physics of Atomic Nuclei 81(5), 569 (2018) 
[13] A.A. Loktionov, A.Kh. Argynova, A.Sh. Gaitinov and T.N. Kvochkina, ISVHECRI (2016). https://doi.org/10.1051/epjconf/201614514004

[14] E.L. Bratkovskaya, W. Cassing, V.P. Konchakovski et al., Journal of Physics: Conference Series 623, 012005 (2015)

[15] A. Motornenko, K. Grebieszkow, E. Bratkovskaya et al., arXiv: 1711.07789 (2018)

[16] W. Broniowski, E. R. Arriola, Phys. Rev. Lett. 112, 112501 (2014)

[17] M. Rybczynski, M. Piotrowski, W. Broniowski, Phys. Rev. C 97, 034912 (2018) 